\title{
The impact of socioeconomic factors on the efficiency of voluntary toxoplasmosis screening during pregnancy: a population- based study
}

\author{
A. E. Lange ${ }^{1 *}$, J. R. Thyrian², S. Wetzka ${ }^{3}$, S. Flessa ${ }^{3}$, W. Hoffmann² ${ }^{2}$ M. Zygmunt ${ }^{4}$, C. Fusch ${ }^{5}$, H. N. Lode
} and M. Heckmann ${ }^{1}$

\begin{abstract}
Background: Congenital toxoplasmosis is associated with severe complications. German state health insurance covers rubella, but not toxoplasmosis, immunity screening. We analysed the effect of socioeconomic factors on the efficiency of private toxoplasmosis screening during pregnancy.

Methods: Toxoplasmosis and rubella screening data ( $n=5402$ mothers) were collected within the population-based Survey of Neonates in Pomerania (SNiP).

Results: At the first-trimester screening, $34.4 \%$ (88.1 \%) of expecting mothers were immune to toxoplasmosis (rubella). Susceptibility for toxoplasmosis (rubella) was observed in $39.6 \%$ (8.9 \%) and $25.8 \%$ (2.95 \%) were not tested. Data on a $2^{\text {nd }}$ screening were available in a subgroup of women with negative immunity showing less than $45 \%$ participation rate. Active toxoplasmosis (no rubella) infection was observed in $0.3 \%(n=17)$ of pregnant women. A multiple logistic regression model $(\mathrm{AIC}=719.67$; $\mathrm{AUC}=0.725)$ revealed that the likelihood of participating in a second toxoplasmosis screening increased among women with a good level of education and a steady partnership and decreased with paternal unemployment and the absence of breastfeeding. The highest probability of non-participation in toxoplasmosis screening was found among women with temporal burden and family responsibilities. A cost-benefit analysis showed that covering general screening for toxoplasmosis with health insurance saved costs.

Conclusion: Toxoplasmosis carried a substantial risk of infection during pregnancy. Although increased socioeconomic status was positively associated with the participation in toxoplasmosis screening, this was not the case when pregnant women had strong temporal burden and family responsibilities. This data supports the need for toxoplasmosis screening among pregnant women as a general healthcare benefit covered by insurance.
\end{abstract}

Keywords: Toxoplasmosis, Screening, Public health, SNiP-Study, Screening pregnancy, Toxoplasmosis infection, Toxoplasmosis screening, Private healthcare services

\footnotetext{
* Correspondence: anja.lange@uni-greifswald.de

1 Department of Pediatrics and Neonatology \& Paediatric Intensive Care,

University Medicine Greifswald, F.-Sauerbruchstr, 17475 Greifswald, Germany

Full list of author information is available at the end of the article
} 


\section{Background}

The protozoan Toxoplasma gondii is a major challenge to public health. The overall rate of toxoplasmosis infection during pregnancy varies from 1 to 120 per 10,000 births, depending on environmental conditions [1,2] Although the German Ministry of Health reported eight cases of acute gestational toxoplasmosis infection in 2009, higher rates seem likely [3].

Following maternal seroconversion, the estimated average risk of fetal infection during pregnancy is $40-50 \%$. Fetal risk correlates inversely with the diaplacental acquisition rate, which increases from approximately $2 \%$ during the first trimester to $30 \%$ during the second trimester, peaking at approximately $80 \%$ immediately before delivery [1, 4-6].

During the first two trimesters, acute toxoplasmosis infection is characterised by septic symptoms, hepatosplenomegaly, thrombocytopenia, hyperbilirubinemia, and central nervous system infections $[1,7,8]$ The latter typically present with encephalitis in combination with retinochorioiditis, hydrocephalus, intracranial calcifications, microphthalmia, and microcephaly, as well as calcifying necroses developing from reactive inflammations, to the point of spontaneous abortion [9]. In contrast, the majority of fetuses infected during the third trimester lack pathological findings at birth (70-90\%) [10, 11]. However, in 30-70\% of offspring with clinical abnormalities, those abnormalities are not detected initially; these children typically have chorioretinitis, hearing loss, and mental retardation later in life $[12,13]$. T. gondii infections are mostly asymptomatic in adults and immunocompetent individuals; consequently, acute infections during pregnancy usually go unnoticed $[8,14]$.

Effective prevention strategies are crucial. One possibility is to provide prophylactic therapy to seroconverted women during pregnancy [15]. When infection is suspected, materno-fetal therapy may be initiated at an early stage. Depending on the gestational week, standardised therapy regimes of different durations markedly alleviate the typical course of toxoplasmosis in neonates $[16,17]$. Knowledge of negative immune status would also enable women to take appropriate preventive precautions [18]. In cases of negative immunity, screenings are conducted at 3-month intervals during gestation to detect possible infections [16, 19].

In Germany, resident gynaecologists offer toxoplasmosis screening and the cost of toxoplasmosis screening is borne by the individual. In contrast, rubella screening is covered by state health insurance, although an anti-rubella vaccine is available. The prevalence of rubella vaccination among German children approaches $75 \%$, depending on where they reside. Similar to toxoplasmosis, the transmission rate of rubella during pregnancy also depends on the time of maternal infection. Sufficient anti-rubella immunity excludes congenital rubella syndrome throughout pregnancy. Women without sufficient immunity are retested later in pregnancy. At the end of pregnancy, a booster vaccine is recommended for mothers with negative immune status [20].

The risk of intrauterine toxoplasmosis infection is higher than that of rubella infection. Less severe disease is commonly reported in countries in which prenatal screening and treatment have been systematically implemented (e.g., France). Gravidic seroconversions (and therefore cases of congenital toxoplasmosis) were reduced in France after toxoplasmosis screening was implemented [21].

Regarding efficacy, there is always the question of to what extent health and monetary concerns can be weighed against each another. A cost-benefit analysis intended to assess the efficacy of a screening program should compare the total cost (the cost of screening and the cost of treatment in cases of seroconversion) with the cost of treatment, rehabilitation, and (in the worst cases) lifelong disability before and after the reduction of cases. Ideally, the screening cost should be equal to or less than the cost of moderating congenital toxoplasmosis.

We used population-based data from the Survey of Neonates in Pomerania (SNiP) to analyse the extent to which toxoplasmosis screening as a privately paid service is used compared with rubella screening (a standard, insurancepaid service), and whether toxoplasmosis screening utilisation correlates with socioeconomic factors.

\section{Methods \\ Study design}

The present study is part of the population-based birth cohort study "Survey of Neonates in Pomerania (SNiP)", conducted from 2002 to 2008. Physicians specially trained for the study collected data about pregnancy and births at the participating hospitals. Detailed information about newborn children and their mothers regarding neonatal health, morbidity, and mortality was collected to calculate prevalence rates for major neonatal diseases, risk factors, and confounding conditions on a crosssectional and prospective basis. According to census data, 7220 babies were born in the study region of Pomerania in northeast Germany during the study period. In SNiP, data from $n=6828$ (95\%) babies and their respective mothers $(n=6747)$ were assessed, yielding high population coverage. Exclusions and non-responders comprised $n=1556$ individuals; a minimum dataset was compiled comprising data on the health status of these newborns and women, but lacking detailed information about environmental parameters. From the mothers, personal data, medical records (149 variables), a personal interview (84 variables), and a self-administered questionnaire concerning socioeconomic background (40 variables) were recorded. Details of the SNiP study have been reported by Ebner et al. [22]. 


\section{Population}

Data from 5402 mothers of neonates delivered between May 2002 and June 2008 were analysed for toxoplasmosis and rubella screening, infection, and socioeconomic background.

\section{Data assessment}

All mothers provided written informed consent to participate in the study, which was approved by the Ethics Committee of the Ernst Moritz Arndt University, Greifswald. Data was collected in standardised 5- to 10-min interviews. Parents were also asked to complete a questionnaire during their stay on the ward and return it to the medical staff before discharge. This questionnaire included questions about the parents' social background and lifestyle. Data about the gestational period and any preventive examinations was acquired using the mother's medical file and maternity card. The collected data was anonymised and stored in an Access database.

Toxoplasmosis and rubella screening data from 5402 mothers was evaluated. This data contained information about immunity against toxoplasmosis and rubella (yes/ no/infection during pregnancy/no information available). Variables were correlated with family status, education level, gainful employment, and income.

To study toxoplasmosis screening attendance as a preventive measure, women were classified into two groups according to whether or not they underwent toxoplasmosis screening. Women who underwent toxoplasmosis screening were stratified into three categories: with positive immunity, defined as proof of IgG antibodies in maternal serum, proof of IgG antibodies in umbilical cord blood, and no evidence of IgM antibodies in umbilical cord blood (Category I); with negative immunity, defined as no proof of IgG antibodies in maternal serum, no proof of IgM antibodies, and no evidence of IgG or IgM antibodies in umbilical cord blood (Category II); and with toxoplasmosis infection during pregnancy, defined as proof of IgM and IgG antibodies in maternal blood or proof of IgM and IgG antibodies in umbilical cord blood (Category III).

For laboratory analysis the Serion Elisa classic Toxoplasmosis gondii IgG/IgM tests (Vision/Serion $\mathrm{GmbH}$, Würzburg, Germany) were used. (Vtox IgG <10UIE) and (vtox IgM $<300$ UIE) demonstrate a negative result. Applying these threshold values result in a sentivity of $98.2 \%(97.8 \%)$ and a specificity of $99.4 \%(95.7 \%)$ for IgG (IgM) (information supplied by the manufacturer). Placental leak occasionally can lead to false-positive IgM reaction. In these cases, the infants were tested again several days later, because the half-life of IgM is only approximately 5 days. A persistent positive or increasing IgG titer in the infant and/or a positive IgM indicated congenital infection. Toxoplasmosis PCR in amniotic fluid was not routinely applied.
In conjunction we use the IgG Avidity Assay. The method is most useful in woman in the first 16 weeks of gestation in whom IgM antibodies are found. It also is useful late in gestation to determine whether infection was acquired 4 or more months earlier, thereby allowing for an estimate of the rate of fetal infection at a given time during gestation [1].

\section{Statistical analysis}

All data were stored using a Microsoft Access 2002 (Microsoft Corporation, Redmond, WA, USA) database. Statistical calculations were performed using SPSS (IBM SPSS 21.0, IBM Deutschland GmbH, Munich, Germany) for Windows $7^{\circ}$ (Microsoft Corporation) and R (R Development Core Team, 2011). The mean (SD), median (range), or numbers of patients were processed for each baseline characteristic and are depicted separately for socioeconomic factors and screening utilisation. Simple logistic regression, with screening outcome as a dependent variable and socioeconomic factors as independent variables, was used to select variables that were associated significantly with screening rate. Subsequently, we evaluated these associations (all variables with $P<0.1$ ) in a multiple logistic regression model (OR $97.5 \% \mathrm{CI})$, taking into account confounders such as the 40 socioeconomic variables mentioned in the Study Design subsection. $P$-values $<0.05$ were considered statistically significant.

\section{Health economic evaluation of general screening}

We developed a simple decision tree (Additional file 1) to estimate whether financial coverage of general toxoplasmosis screening by health insurance would be beneficial. We compared the expected value of total direct and indirect costs for two scenarios (represented in the two primary branches of the decision tree). Scenario 1 represented complete coverage of screening costs by health insurance, resulting in higher screening participation and a correspondingly lower number of cases. Scenario 2 represented the status quo in Germany (screening paid for out-of-pocket). The main input variables of the model were the costs of screening, treatment, and indirect costs (loss of lifelong productivity), as well as the likelihoods of screening (subject to scenarios 1 and 2), toxoplasmosis, treatment, and death. Data was obtained from literature [23] and adjusted using findings from SNiP.

\section{Results}

\section{Study population}

For all pregnant women $(n=5402)$, utilisation rate for early rubella screening as a standard service was $97.05 \%$; the utilisation rate for toxoplasmosis screening as a private service was $74 \%$ (Fig. 1). 


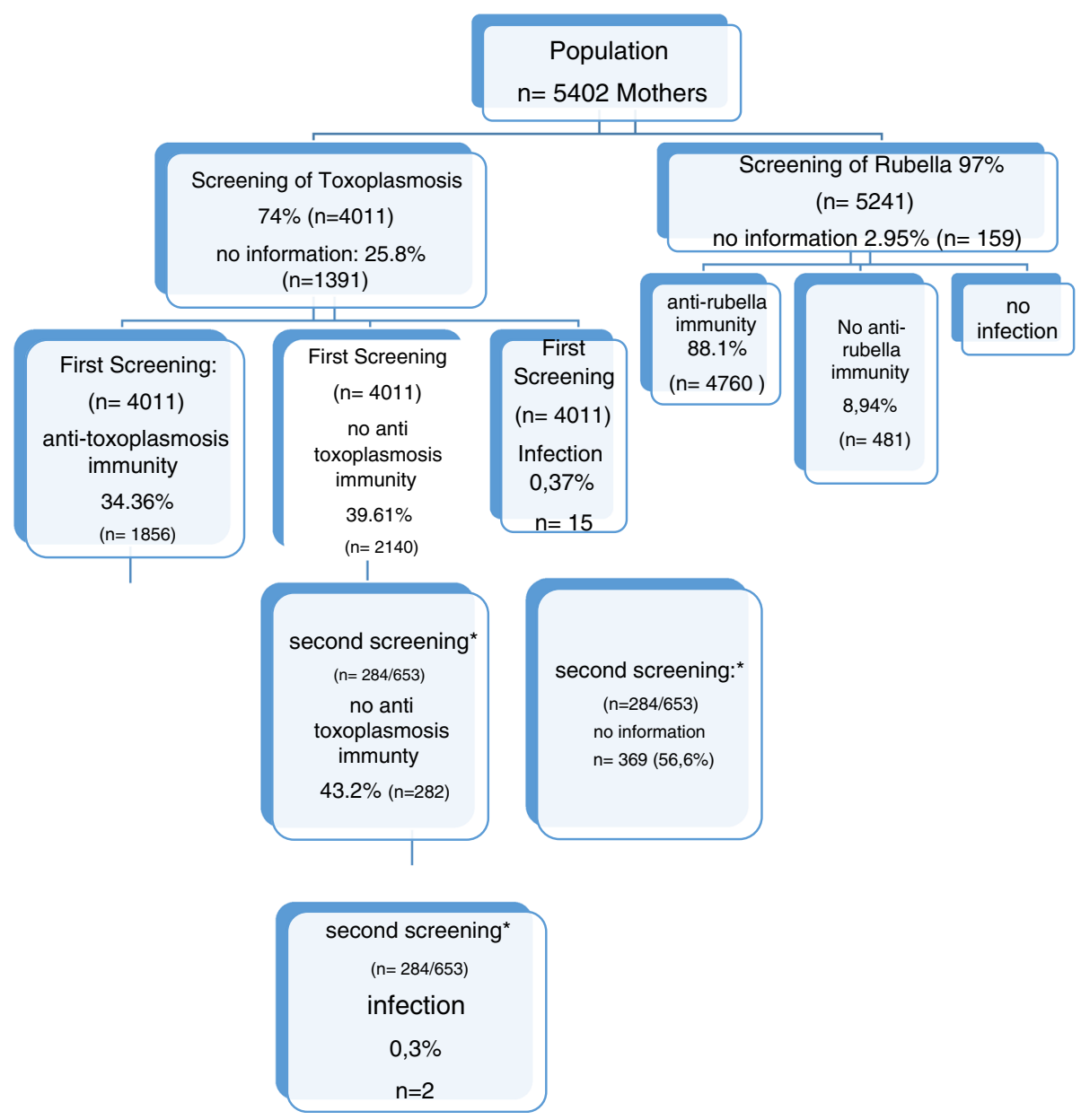

Fig. 1 Participation of pregnant women in toxoplasmosis and rubella screening. Data on a 2nd screening were available in a subgroup of women from 2002-2003

\section{Screening results: toxoplasmosis vs. rubella immunity First screening}

At the first screening, the rate of anti-toxoplasmosis immunity $(n=1856 ; 34.36 \%)$ was less than one-half the rate of anti-rubella immunity $(n=4760 ; 88.11 \%)$. Screening revealed 2140 cases $(39.61 \%)$ that lacked toxoplasmosis immunity and 481 cases $(8.94 \%)$ that lacked rubella immunity. Data from the mothers' medical files and maternity cards provided no information regarding toxoplasmosis immunity status for 1391 women $(25.8 \%)$ and regarding rubella immunity status for 159 women ( $2.95 \%$ ). Active toxoplasmosis infection during pregnancy was observed in 17 women ( $0.3 \%$, CI 95 0.2- 0.6). Infection occurred during pregnancy at gestational age of up to 10 weeks in $n=6(35.3 \%)$ cases, in week 11-18 in $n=6$ (35.3\%), in 3 cases there were no information (Table 1). The mean gestational age of their newborns was 40.3 weeks $(\mathrm{SD}=1.01)$. There was suspicious sonography of the skull in $n=2(11.76 \%)$ newborns and no hearing deficit in any of the newborns (neither right or left ear). Active infection was detected in cord blood from eight newborns (Table 1).

No active rubella infections were documented.

\section{Second screening}

Six hundred fifty-three women who initially screened negative for toxoplasmosis were offered a second screening from May 2002 to March 2003; 284 (43.5 \% of $n=653$ ) declined a second screening (Fig. 1). Two hundred eightytwo women $(43.2 \%)$ had a negative screening result; two $(0.31 \%)$ had seroconverted since the first screening, indicating infection during early pregnancy.

\section{Impact of socioeconomic factors on screening participation: univariate analysis}

Details of the univariate analysis are available online as Supplementary Information. 
Table 1 Treatment and neonatal short-term outcome of 17 Pregnancies with maternal toxoplasma infection. SGA small for gestational age

\begin{tabular}{|c|c|c|c|c|c|c|c|c|c|}
\hline Newborn & $\begin{array}{l}\text { GA AT infection } \\
\text { (weeks) }\end{array}$ & $\begin{array}{l}\text { Mother } \\
\text { IGM }\end{array}$ & $\begin{array}{l}\text { Mother } \\
\text { IGG }\end{array}$ & Avidity & $\begin{array}{l}\text { Treatment } \\
\text { spiramycin }\end{array}$ & $\begin{array}{l}\text { Newborn } \\
\text { IGG }\end{array}$ & $\begin{array}{l}\text { Newborn } \\
\text { IGM }\end{array}$ & Findings in the fetus & $\begin{array}{l}\text { Treatment } \\
\text { newborn }\end{array}$ \\
\hline 1 & 6 & + & + & no & + & - & - & no & no \\
\hline 2 & 8 & + & + & no & + & - & - & no & no \\
\hline 3 & 8 & + & + & low & + & + & - & SGA & yes \\
\hline 4 & 8 & + & + & low & + & + & $t^{a}$ & no & yes \\
\hline 5 & 10 & + & + & low & $?$ & + & - & no & no \\
\hline 6 & 10 & + & + & low & - & $\begin{array}{l}+ \\
\text { lgG } \\
\text { increased }\end{array}$ & - & $\begin{array}{l}\text { Cerebral ventricular } \\
\text { dilatation }\end{array}$ & yes \\
\hline 7 & 11 & + & + & low & + & + & - & no & no \\
\hline 8 & 12 & + & + & low & inadequate & - & - & SGA & no \\
\hline 9 & 12 & + & + & low & + & + & $t^{a}$ & SGA & yes \\
\hline 10 & 14 & + & + & high & + & $\begin{array}{l}+ \\
-{ }_{-}^{a}\end{array}$ & - & no & no \\
\hline 11 & 16 & + & + & low & - & + & + & Cerebral Disorders & yes \\
\hline 12 & 17 & + & + & low & + & + & - & no & no \\
\hline 13 & No informatio & + & + & low & + & + & - & no & no \\
\hline 14 & No information & + & + & low & No information & + & - & no & no \\
\hline 15 & No information & + & + & low & No information & + & - & no & no \\
\hline $16^{b}$ & 20 & + & + & no & - & + & + & SGA & yes \\
\hline $17^{b}$ & 21 & + & + & no & - & + & + & SGA & yes \\
\hline
\end{tabular}

at repeated blood sampling, ${ }^{\mathrm{b}}$ Newborn 16 and 17 : detected at the second screening

\section{Correlation of family status with first screening participation}

Participation in the first toxoplasmosis screening correlated significantly with the family status of pregnant women $(n=3999, p>0.001)$. Married pregnant women living with a spouse had the highest participation rate (74.5\%); married women separated from a spouse had a $60 \%$ participation rate (Additional file 2: Table S1). Single pregnant women participated in the first toxoplasmosis screening at a much higher rate $(75.5 \%)$ than married pregnant women not living with a spouse $(60.0 \%)$ or divorced pregnant women (59.8\%).

In contrast, we observed no statistically significant correlation between participation in rubella screening and family status ( $n=3999$ [62.91 \% of the 5402 women included in analysis], $p=0.42$ ). Married women and married women living separated from a spouse took part in rubella screening at high rates (97.3 and $91.1 \%$, respectively). Rubella screening participation was also high among single (97.9\%), divorced (98.3\%), and widowed (100\%) women.

\section{Correlation of patient-reported education with first screening participation}

Education level and participation in toxoplasmosis and rubella screenings correlated positively $(n=4813$ [89.1\% of the 5402 women included in analysis], $p<0.001)$. Mothers who had 8 years of secondary school and were universityqualified (Abitur, 83.4\%), had 8 years of secondary school (Fachhochschulreife, similar to A-levels, $78.50 \%$ ), and had 6 years of secondary school (Realschulabschluss, $76 \%$ ) participated more often in toxoplasmosis screening than mothers who had 5 years of secondary school (Hauptschulabschluss, $61.1 \%$ ), did not earn a school diploma (47.6\%), or were still in school (44.4\%). Participation in rubella screening was high across all educational levels (Additional file 3: Table S2).

\section{Correlation between income level and first screening participation}

Expectant mothers' income level correlated positively with participation in the first toxoplasmosis screening ( $n=2938$ [54.38 \% of the 5403 women included in the analysis], $p<0.001$ ) (Additional file 4: Table S3). This correlation was not observed for rubella screening.

\section{Influence of socioeconomic factors on participation in the second toxoplasmosis screening}

Attendance at the second toxoplasmosis screening was higher among women with a higher education level and 
women who had been gainfully employed before pregnancy (Additional file 5: Table S4).

\section{Impact of socioeconomic factors on first screening participation: multiple logistic regression analysis}

We calculated a multiple logistic regression model to evaluate the associations (all variables with $p<0.1$ ) between screening participation and each of the studied socioeconomic factors (family status, education level, income level, steady partnership, gainful employment, planned pregnancy, and breastfeeding) (OR $97.5 \% \mathrm{CI}$; Table 2). The model demonstrated a modest fit (AIC = 719.67; AUC $=0.725$ ). When socioeconomic factors were viewed as cofounders, the likelihood of participating in a second toxoplasmosis screening increased among women with a good level of maternal education and a steady partnership. The likelihood of non-participation in an adequate toxoplasmosis screening increases by 1.14 with paternal unemployment and by 1.72 in the absence of breastfeeding.

Table 3 shows the probability of non-participation in toxoplasmosis screening when socioeconomic variables were combined in different ways. The highest probability of non-participation (66 \%) was found in pregnant women with a high educational level, full-employed, planned their pregnancy, did not intend to breast-feed and lived in a steady partnership with an unemployed partner.

\section{Health economic evaluation of general screening}

Based on the decision tree model (Fig. 1) and the data provided by [23], we estimated the expected lifelong cost as $\$ 633$ USD for full coverage by health insurance and \$1406 USD for out-of-pocket payment. From a societal perspective, coverage of toxoplasmosis screening by health insurance is advisable.

\section{Discussion}

Substantial pathology in newborns, insufficient screening strategies, and the lack of a vaccine contribute to $T$. gondii as a considerable public health concern. In our cohort, sufficient immunity was detected in only $34.4 \%(n=1856)$ of mothers-to-be; $25.8 \%(n=1391)$ of women were never screened, and approximately $40 \%(n=2140)$ were initially diagnosed as lacking immunity without sufficient followup. The latter population urgently requires counselling regarding preventative measures and re-screening. In contrast, $88 \%(n=4760)$ of pregnant women were effectively protected against rubella. To avoid potential gestational toxoplasmosis infection, the immune status of pregnant women should be monitored at 3-month intervals [8]. But even worse, less than $45 \%$ of women without immunity to toxoplasmosis participated in a second screening.

Our prevalence data indicate a gestational infection rate of $0.3 \%(n=17 / 5402)$, and active infection was detected in cord blood from eight newborns (8/3645; $0.22 \%)$. These numbers are considerably higher than the recently published report that 8.5 in 10,000 pregnant women have toxoplasmosis, and 1.0 per 10000 infants had congenital toxoplasmosis $(13 \%$ mean transmission rate) in Austria [24]. While in Austria toxoplasmosis screening in pregnancy is covered by national healthcare providers, this is not the case in Germany. The Law on the Prevention of infection provides anonymous mandatory reporting to the Robert-Koch-Institute (German Ministry of Health). But only 11 cases of active congenital toxoplasmosis were reported in 2008, [3] modelled calculations based on our study data yielded much higher rates, in agreement with other published calculations [8]. Furthermore, congenital toxoplasmosis often goes unreported; cases may not be detected until patients experience manifestations as adults [25].

A retrospective analysis at the Ludwig-MaximillianUniversity in Munich (LMU) reported on toxoplasmosis screening in 15,856 pregnancies between 2001 and 2008 $[26,27]$. Toxoplasmosis serology was completed in $39.99 \%$; of these, $62.47 \%$ were seronegative and $13.02 \%$ were seropositive. Seroconversion was discovered in $0.14 \%$ of these women, resulting in a calculated incidence of $<0.057 \%$. This difference in immunity level may be explained by geography: LMU patient population is urban, while participants in the SNiP study live in

Table 2 Impact of socioeconomic factors on first screening participation: multiple logistic regression analysis with non-participation in toxoplasmosis screening as the dependent variable

\begin{tabular}{llccc}
\hline & OR & Cl 2.50\% & $97.5 \%$ Sign test $p$-value & 0.78 \\
Solid partnership (=yes) & 0.39 & 0.18 & 0.80 & $0.0115^{*}$ \\
Maternal education level (=high) & 0.68 & 0.57 & 1.25 & $0.000^{* * *}$ \\
Job situation, mother (=employed) & 1.14 & 1.03 & 1.14 & $0.0095^{* *}$ \\
Job situation, father (=employed) & 0.86 & 0.69 & 1.79 & $0.0487^{*}$ \\
Planned pregnancy & 1.4 & 1.10 & 2.18 & $0.0071^{* *}$ \\
Absence of breastfeeding & 1.7 & 1.35 & $0.000^{* * *}$ \\
\hline
\end{tabular}

${ }^{a} n=3399$ women $(62.91 \%$ of the 5402 included in the analysis)

${ }^{* * *} p<0.001 ;{ }^{* *} p<0.01 ;{ }^{*} p<0.05$ 
Table 3 Probability of non-participation in toxoplasmosis screening

\begin{tabular}{|c|c|c|c|c|c|c|}
\hline $\begin{array}{l}\text { Solid } \\
\text { partnership }\end{array}$ & $\begin{array}{l}\text { Education level, } \\
\text { mother }\end{array}$ & $\begin{array}{l}\text { Job situation, } \\
\text { mother }\end{array}$ & $\begin{array}{l}\text { Job situation, } \\
\text { father }\end{array}$ & $\begin{array}{l}\text { Planned } \\
\text { pregnancy }\end{array}$ & Breastfeeding & $\begin{array}{l}\text { Probability of non- } \\
\text { participation }\end{array}$ \\
\hline Yes $^{a}$ & Yes $^{b}$ & Full Time & No education & Yes & No & $66.01 \%$ \\
\hline Yes & Yes & Full Time & No education & Yes & No & $62.92 \%$ \\
\hline Yes & Yes & Unemployed & At school & Yes & No & $61.97 \%$ \\
\hline Yes & Yes & Unemployed & At school & Yes & No & $58.95 \%$ \\
\hline Yes & Yes & Unemployed & No education & Yes & Yes & $55.42 \%$ \\
\hline Yes & Yes & Full Time & At school & No & No & $54.76 \%$ \\
\hline Yes & Yes & Full Time & At school & No & No & $54.76 \%$ \\
\hline
\end{tabular}

${ }^{a} n=4813$ women (89.1 \% of the 5402 included in the analysis)

${ }^{b}$ mother in a stable partnership

${ }^{c}$ mother has completed a professional education

Mecklenburg-Western Pomerania. In the latter state, which is dominated by farming communities, women are exposed to a greater environmental risk of contracting toxoplasmosis (seropositive: SNiP $34.36 \%$ vs. LMU 13.02 \%; seronegative: SNiP 39.61 \% vs. LMU 62.47 \%).

Each screening is subject to a fee that is paid by the individual [25]. Therefor, the current screening strategy (screening as a private service) may be one reason for the insufficient number of toxoplasmosis screenings. Although one screening during pregnancy is sufficient for women with positive immune status, cases that lack immunity require three examinations to identify gestational infections in adequate time to initiate materno-fetal therapy [25]. We contrast this situation against rubella screening, a standard service covered by state health insurance. The rate of maternal participation is $23 \%$ higher for rubella screening than for toxoplasmosis screening, although vaccination renders the rate of rubella immunity $(88.11 \%)$ much higher than that of toxoplasmosis immunity (34.36 \%).

Socioeconomic factors also have a marked negative influence on the rate of screening participation. We have demonstrated that attendance at toxoplasmosis screenings is higher among mothers with higher education levels and gainful employment preceding pregnancy.

In this study, lower socioeconomic status was a clear risk factor for non-participation in toxoplasmosis screening. Toxoplasmosis is more likely to occur among neonates born to mothers in the lower social strata. Although increased socioeconomic status was positively associated with the participation in toxoplasmosis screening, this was not the case when pregnant women had strong temporal burden and family responsibilities. Our model demonstrated that mothers who are in a long-term relationship, have graduated from school, are working full-time, with a partner who is not working full-time (student, in training), undergoing an intended/planned pregnancy, and with no intention of breastfeeding are least likely to make use of toxoplasmosis screening programs (Table 3). These effects may not be observed as strongly in rubella screenings as the costs for this are covered by public insurance, whereas toxoplasmosis screenings are not and thus also require additional consulting with the responsible physicians. This is associated with more time having to be contributed by the mother, which may have aggravated the negative effect on participation rate in toxoplasmosis screening.

Our analysis shows that the expected lifelong cost for out-of-pocket payment are 2.2 fold the cost for full coverage by health insurance, i.e., the society could save 773 USD per pregnancy if the health insurance had the obligation to pay for screening for toxoplasmosis. This analysis is preliminary as some potential arms of the decision tree (Fig. 1) are not included, such as false positive sero-conversions with unnecessary amniocentesis and fetal loss. However, with a ratio of 2.2 the results are quite robust and allow the general statement that screening is cost-effective. This finding is in line with prior findings that general toxoplasmosis screening is highly cost-efficient from a societal perspective [23], but more research on the economics of toxoplasmosis screening is needed to address the complete complexity of the epidemiological, economic and social situation.

Stillwaggon et al. [23] we highlight a general dilemma of healthcare financing: cost-effectiveness of general screening from a societal perspective does not ensure implementation by health insurance. The high figures provided by Stillwaggon et al [23] include indirect costs (i.e., lifelong loss of productivity, fatalities), which are irrelevant from the perspective of health insurance. The asymmetry of societal and institutional benefits regularly requires legal action (e.g., the obligatory financing of toxoplasmosis screening by health insurance). Our findings also warrant additional research regarding the health economics of so-called neglected diseases (Fig. 1).

Because the risk of toxoplasmosis infection is higher in mothers-to-be with negative immune status, they should be screened three times during pregnancy to detect potential seroconversion as early as possible. In our study we found 15 cases of toxoplasmosis out of 4011 woman at the first screening, which means that 267 
mothers were screened to detect one case of toxoplasmosis. Furthermore, the rate of mothers with maternal toxoplasmosis who were treated to prevent one treatment in the offspring was 9/3 (Table 1) [28]. In France, this screening strategy reduced congenital toxoplasmosis cases by 10-fold [21]. Furthermore, the Austrian toxoplasmosis register reported recently that early detection of toxoplasmosis in pregnancy allowed adequate treatment with a 6.fold reduction in transmission rate [24].

In our cohort, participation has to be regarded as inadequate during toxoplasmosis screenings at all stages.

A limitation of our study is the lack of serologic follow up data of newborns at risk during the first year of life. A second limitation is that data on the acceptance of a $2^{\text {nd }}$ screening derived only from a subgroup of our cohort. These limitations may have resulted in a higher incidence of both toxoplasmosis infections in pregnancy and in the newborn in our population.

In conclusion, private toxoplasmosis screening in pregnancy carries a substantial risk of undiagnosed infection. Participation in toxoplasmosis screening was influenced by socioeconomic factors. Increased socioeconomic status was positively associated with the participation in toxoplasmosis screening. But this was not the case when pregnant women had strong temporal burden and family responsibilities. It seems reasonable to make toxoplasmosis screening a standard of care and a reimbursable healthcare benefit for all pregnant women, as our data show that large numbers of pregnant women do not participate adequately in this screening. These considerations reflect the urgent need for a globally applicable and consistent toxoplasmosis screening strategy for pregnant women. Such a strategy could improve awareness, knowledge, and prevention, and could guarantee early treatment in cases of gestational infection. Taking into account the potential follow-up costs for neonates with undiagnosed toxoplasmosis infections, health economic considerations may also support the general screening of all women while trying to conceive and/or during early pregnancy.

\section{Conclusion}

Toxoplasmosis carried a substantial risk of infection during pregnancy. Although increased socioeconomic status was positively associated with the participation in toxoplasmosis screening, this was not the case when pregnant women had strong temporal burden and family responsibilities. This data supports the need for toxoplasmosis screening among pregnant women as a general healthcare benefit covered by insurance.

Taking into account the potential follow-up costs for neonates with undiagnosed toxoplasmosis infections, health economic considerations may also support the general screening of all women while trying to conceive and/or during early pregnancy.

\section{Additional files}

Additional file 1: A Simple Decision Tree Model of Health Economic Evaluation of General Toxoplasmosis Screening. (TIF 612 kb)

Additional file 2: Table S1. Correlations between family status and participation in first toxoplasmosis and rubella screenings ${ }^{a} n=4813$ women (89.1\% of the 5402 included in the analysis). ${ }^{* *} p<0.001 ;{ }^{*} p=0.42$ regarding participation vs. lack of participation. All data are presented as percentages. (DOCX $11 \mathrm{~kb}$ )

Additional file 3: Table S2. Participation in first toxoplasmosis and rubella screenings with respect to education level, $n=4813$ (89.1\% of 5402) women included in the analysis. All data are presented as percentages. ${ }^{*} p<0.05 ;{ }^{* *} p<0.01 ;{ }^{* * *} p<0.001 ;{ }^{* * * *} p<0.0001$. (DOCX $12 \mathrm{~kb}$ )

Additional file 4: Table S3. Participation in first toxoplasmosis or rubella screening with respect to income level, $n=4813$ (89.1\% of 5402) women included in the analysis. All data are presented as percentages. ${ }^{*} p<0.05 ;{ }^{* *} p<0.01$; ${ }^{* * *} p<0.001 ;{ }^{* * * *} p<0.0001$. (DOCX $11 \mathrm{~kb}$ )

Additional file 5: Table S4. Influence of socioeconomic factors on participation in second toxoplasmosis screening, $n=4813$ (89.1 \% of 5402) women included in the analysis. All data are presented as percentages. ${ }^{*} p<0.05$; ${ }^{* *} p<0.01$; ${ }^{* * *} p<0.001$; ${ }^{* * *} p<0.001$. (DOCX $12 \mathrm{~kb}$ )

\section{Abbreviations}

AIC, Area in the curve; AUC, Area under the curve; $\mathrm{Cl}$, Confidence interval; e.g, for example; IgG, Immunoglobulin G; IgM, Immunglobuline M; LMU, Ludwig Maxilmilian Universtiy Munich; OR, Odds ratio; PCR, polymerase change reaction; SD, standard deviation; SNIP, Survey of Neonates in Pomerania; T.gondii, Toxoplasma gondii; UIE, unit international; USD, United States Dollar.

\section{Acknowledgements}

This work is part of the Research Cooperation Community Medicine (RCC) of the University of Greifswald, Germany, which is funded by the Federal Ministry of Education and Research (grant no. ZZ 96030). The RCC encompasses several research projects that share data obtained through population-based studies (http://www.community-medicine.de). SNiP was supported by the German Federal Ministry for Education and Research (NBL3 program, grant 01 ZZ 0103), the Excellence Support Programme of the Federal State of Mecklenburg-Western-Pomerania (UG 07 034) "Genetics of frequent variants in INSIG2, their role in the pathogenesis of obesity and other disorders particularly in children", and the Epidemiological Study on Childhood Cancer and Malformations in the Vicinity of Nuclear Power Plants (Stsch 4493). The Medical Faculty of the Ernst Moritz Arndt University of Greifswald provided additional support in the form of a training grant to AL.

This study was supported by a grant from the German Ministry for Education and Research to CF (NBL3: 01 ZZ 0403).

\section{Funding}

SNiP was supported by (a) the German Federal Ministry for Education and Research (NBL3 program, grant 01 ZZ 0103), (b) the Excellence Support Programme of the Federal State of Mecklenburg-Vorpommern (UG 07034 ) 'Genetics of frequent variants in INSIG2, their role in the pathogenesis of obesity and other disorders particularly in children' awarded to D. Rosskopf and J. P. Haas, (c) the Epidemiological Study on Childhood Cancer and Malformations in the Vicinity of Nuclear Power Plants (StSCH 4493).

\section{Availability of data and materials}

The data of the SNIP-study is publicly available via https://www.fvem.med.uni-greifswald.de/dd_service/data_use_intro.php?lang=ger. This is a data repository where any researcher can register and find data dictionary as well as an online application tool for getting access to data. Upon an application by registered users, the Research Cooperation Community Medicine (RCC) of the University of Greifswald, Germany, which is funded by the Federal Ministry of Education and Research (grant no. ZZ 96030) decides on granting access to the data based on scientific guidelines. 


\section{Authors' contributions}

AEL was responsible for data collection and writing of the manuscript and JRT was responsible in data collection and quality assessment, SW and SF developed the Simple Decision Tree Model of Health Economic Evaluation of General Toxoplasmosis Screening, MZ, HNL and CF was responsible for quality assessment and revising the manuscript, $\mathrm{WH}$ and $\mathrm{MH}$ originally conceived of the study and contributed to scientific interpretations and manuscript revision. The authors declare that they have no competing interest. I confirm that all authors have read and approved submission of the manuscript and that material in the manuscript has not been published and is not being considered for publication elsewhere in whole or in part in any language or any form.

\section{Competing interests}

The authors declare that they have no competing interests.

\section{Consent for publication}

Not Applicable.

\section{Ethics approval and consent to participate}

Research based on patient-related data and human DNA is strictly regulated by German law. The collection of detailed personal data combined with sampling of biomaterials demands strict confidentiality. Our study complies with international guidelines of ethical research based on the Declaration of Helsinki. The study design was reviewed and approved by the Ethics Committee of the Board of Physicians Mecklenburg-Western Pomerania at the University of Greifswald. Eligible women were asked for written informed consent; in cases of legally minor mothers, i.e. aged $<18$ years, the additional signature of the newborn's legal care-giver was required. Written informed consent included data assessment in face-to-face interviews, self-administered questionnairs and patient records. It also covered biosamples of blood. Furthermore, data storage of pseudonomized data was given informed consent to, as well as their analyses and publication.

\section{Author details}

'Department of Pediatrics and Neonatology \& Paediatric Intensive Care, University Medicine Greifswald, F.-Sauerbruchstr, 17475 Greifswald, Germany. ${ }^{2}$ Division of Health Care Epidemiology and Community Health, Institute of Community Medicine, University of Greifswald, Greifswald, Germany. ${ }^{3}$ Department of Health Care Management, Faculty of Law and Economics, Ernst-Moritz-Arndt-University of Greifswald, Greifswald, Germany.

${ }^{4}$ Department of Gynaecology and Obstetrics, University of Greifswald, Greifswald, Germany. ${ }^{5}$ Division of Neonatology, Department of Pediatrics, McMaster University, Hamilton, ON, Canada.

Received: 8 November 2015 Accepted: 12 July 2016

Published online: 29 July 2016

\section{References}

1. Remington \& Klein, Wilson \& Baker, Infectious Diseases of the Fetus and Newborn Infant, 2006 sixth Edition:31:948- 1091

2. Tenter A, Heckeroth AR, Weiss LM. Toxoplasma gondii: from animals to humans. Int J Parasitol. 2000;30:1217-58.

3. Robert-Koch-Institut. Infectionsepidemiologisches Jahrbuch 2008.

4. Berrebi A, Bardou M, Bessieres MH, Nowakowska D, Castagno R, Rolland M, et al. Outcome for children infected with congenital toxoplasmosis in the first trimester and with normal ultrasound findings: a study of 36 cases. Eur J Obstet Gynecol Reprod Biol. 2007;135(1):53-7. Epub 2006/12/27. eng.

5. Hill D, Dubey JP. Toxoplasma gondii: transmission, diagnosis and prevention. Clin Microbiol Infect. 2002:8(10):634-40. Epub 2002/10/23. eng.

6. Robert-Gangneux F, Gavinet MF, Ancelle T, Raymond J, Tourte-Schaefer C, Dupouy-Camet J. Value of prenatal diagnosis and early postnatal diagnosis of congenital toxoplasmosis: retrospective study of 110 cases. J Clin Microbiol. 1999;37(9):2893-8. Pubmed Central PMCID: 85406. Epub 1999/08/17. eng.

7. Hedriana HL, Mitchell JL, Brown GM, Williams SB. Normal fetal outcome in a pregnancy with central nervous system toxoplasmosis and human immunodeficiency virus infection. A case report. J Reprod Med. 1993;38(9):747-50.

8. Cristoph J, Kattner E, Seitz HM, Reiter-Owona I. Strategien zur Diagnostik und Behandlung der pränatalen Toxoplasma-Infektion - ein aktueller Überblick. Z Geburtshilfe Neonatologie. 2004;208:10-6.
9. Montoya JG, Liesenfeld O. Toxoplasmosis. Lancet. 2004;363(9425):1965-76. Epub 2004/06/15. eng.

10. Lebech M, Joynson DH, Seitz HM, Thulliez P, Gilbert RE, Dutton GN, et al. Classification system and case definitions of Toxoplasma gondii infection in immunocompetent pregnant women and their congenitally infected offspring. European Research Network on Congenital Toxoplasmosis. Eur J Clin Microbiol Infect Dis. 1996;15(10):799-805.

11. Mombro M, Perathoner C, Leone A, Buttafuoco V, Zotti C, Lievre MA, et al. Congenital toxoplasmosis: assessment of risk to newborns in confirmed and uncertain maternal infection. Eur J Pediatr. 2003;162(10):703-6.

12. Vutova K, Peicheva Z, Popova A, Markova V, Mincheva N, Todorov T. Congenital toxoplasmosis: eye manifestations in infants and children. Ann Trop Paediatr. 2002;22(3):213-8

13. Safadi MA, Berezin EN, Farhat CK, Carvalho ES. Clinical presentation and follow up of children with congenital toxoplasmosis in Brazil. Braz J Infect Dis. 2003;7(5):325-31.

14. Dunn D, Wallon M, Peyron F, Petersen E, Peckham C, Gillbert R. Mother-tochild transmission of toxoplasmosis: risk estimates for clinical counselling. Lancet. 1999;353(9167):1829-33. Epub 1999/06/08. eng.

15. Pfaff AW, Candolfi E. New insights in toxoplasmosis immunology during pregnancy. Perspective for vaccine prevention. Parassitologia. 2008:50(1-2):55-8.

16. Roberts F, Roberts CW, Ferguson DJ, McLeod R. Inhibition of nitric oxide production exacerbates chronic ocular toxoplasmosis. Parasite Immunol. 2000;22(1):1-5.

17. Robert-Koch-Instiut. Infektionsepidemiologisches Jahrbuch,Toxoplasmose 2009 Global Public Health. Berlin, Heidelberg, New York: Springer; 2009.

18. Elsheikha HM. Safer food for pregnant women: Practices and risks. Public Health. 2008;122(12):1407-9. doi:10.1016/j.puhe.2008.06.002. Epub 2008 Sep 7.

19. Dollfus H, Dureau P, Hennequin C, Uteza Y, Bron A, Dufier JL. Congenital toxoplasma chorioretinitis transmitted by preconceptionally immune women. Br J Ophthalmol. 1998;82(12):1444-5. Pubmed Central PMCID: 1722451.

20. Best JM. Rubella. Semin Fetal Neonatal Med. 2007;12(3):182-92. Epub 2007/03/06 eng.

21. Ambroise-Thomas P, Schweitzer M, Pinon JM, Thiebaugeorges O. Prevention of congenital toxoplasmosis in France. Risk assessment. Results and perspectives of prenatal screening and newborn follow up. Bull Acad Natl Med. 2001;185(4):665-83. discussion 84-8. La prevention de la toxoplasmose congenitale en France. Evaluation des risques. Resultats et perspectives du depistage antenatal et du suivi du nouveau-ne.

22. Ebner A, Thyrian JR, Lange A, Lingnau ML, Scheler-Hofmann M, Rosskopf D, et al. Survey of Neonates in Pomerania (SNiP): a population-based birth study-objectives, design and population coverage. Paediatr Perinat Epidemiol. 24(2):190-9. Epub 2010/04/27. eng.

23. Stillwaggon E, Carrier CS, Sautter M, McLeod R. Maternal serologic screening to prevent congenital toxoplasmosis: a decision-analytic economic model. PLoS Negl Trop Dis. 2011;5(9):e1333.

24. Prusa AR, Kasper C, Pollak A, Gleiss A, Waldhoer T, Hayde The Austrian Toxoplasosis Register, 1992- 2008. Clin. Infect Dis.2014 Sep 12. Pii:ciu 724. [Epub ahead of print]

25. Gross U. Prevalence and public-health-aspects of toxoplasmosis. Bundesgesundheitsblatt Gesundheitsforschung Gesundheitsschutz. 2004; 47(7):692-7. Pravalenz und Public-Health-Aspekte der Toxoplasmose.

26. Abboud P, Villena I, Chemla C, Leroux B, Talmuld M, Bednarczyk L, et al. Screening for congenital toxoplasmosis: pregnancy outcome after prenatal diagnosis in 211 cases. J Gynecol Obstet Biol Reprod. 1997;26(1):40-6. Depistage de la toxoplasmose congenitale: devenir des grossesses apres le diagnostic antenatal. A propos de 211 cas.

27. [Comment on the publication by Abholz, H. H.: Screening for toxoplasmosis in pregnancy: more risk than benefit]. Gesundheitswesen. 1994 Jul;56(7):4112. Zur Publikation Abholz, H.H.: Toxoplasmose-Screening in der Schwangerschaft: mehr Schaden als Nutzen.

28. Robert- Gangneux F, et al. It is not only the cat that did it: how to prevent and treat congenital toxoplasmosis. Infect. 2014;68 Suppl 1: S125-33. doi:10.1016/j.jinf.2013.09.023. Epub 2013. 